\title{
Multiple ionization of rare gases by hydrogen-atom impact
}

\author{
L. Sarkadi, ${ }^{*}$ P. Herczku, S. T. S. Kovács, and Á. Kövér \\ Institute for Nuclear Research, Hungarian Academy of Sciences (MTA Atomki), P.O. Box 51, H-4001 Debrecen, Hungary
}

(Received 12 April 2013; published 12 June 2013)

\begin{abstract}
Cross sections for the multiple ionization of $\mathrm{He}, \mathrm{Ne}, \mathrm{Ar}$, and $\mathrm{Kr}$ by $\mathrm{H}^{0}$ impact with and without the simultaneous ionization (electron loss) of the projectile are presented in the energy range $75-300 \mathrm{keV}$. The data were measured by coincident detection of the recoil target ions and the charge-state analyzed scattered projectiles. To obtain information about the role played by the electron of $\mathrm{H}^{0}$ in the collision, the measurements were repeated with protons under the same experimental conditions. The measured data are analyzed using the classical trajectory Monte Carlo (CTMC) method. CTMC describes well the experimental data for both projectiles for single vacancy creation; however, increasing deviation is observed between theory and experiment with increasing number of created vacancies and with decreasing target atomic number.
\end{abstract}

DOI: 10.1103/PhysRevA.87.062705

PACS number(s): $34.50 . \mathrm{Fa}$

\section{INTRODUCTION}

Understanding of the mechanisms of collisions between energetic charged particles and neutral atoms is of fundamental importance. Furthermore, the results achieved in this field significantly contribute to the development of many research areas (plasma physics, astrophysics, materials science, etc.), and they find numerous applications in practice.

The subject of the present work is the multiple ionization of atoms. This is a very complex process even for the simplest, structureless projectiles (protons, antiprotons, electrons, positrons, alpha particles, etc.). For a bare, positively charged particle there are many reaction channels that may contribute to the total multiple ionization cross section: direct multiple electron ejection (ionization), charge transfer with possible simultaneous additional ionization, and resonant electron emission following double outer-shell excitation (autoionization) or inner-shell ionization (Auger decay). The relaxation of the atoms excited or ionized in their inner shells can produce additional vacancies via Auger cascades. The inner-shell decay processes (emission of $\mathrm{x}$ rays or Auger electrons) are often accompanied by the ejection of shake-off electrons. For electron impact the understanding of the process is simplified by the lack of the charge transfer channel, but, at the same time, the electron exchange effect has to be considered, particularly at low collision energies. The simplest and cleanest dynamics occurs at antiproton impact: in this case the only reaction channel is pure ionization. A comprehensive overview of multiple ionization induced by antiprotons, protons, and electrons in rare gases has been published recently by Montanari et al. [1].

The complexity of the multiple ionization processes increases for structured projectiles. In this case the most interesting question concerns the role played by the constituents of the projectile in the collision. Such structured particles are partially stripped ions, neutral atomic projectiles, and an exotic particle that has been used only recently in collision experiments, the positronium. In a simplified picture the role of the electrons in these composite particles can be either passive or active. The passive role means that the electrons exert only

\footnotetext{
*sarkadil@atomki.hu
}

a screening effect on the Coulomb field of the nucleus of the projectile ion (atom). This is mostly characteristic of deeply bound electrons. At a high enough energy, weakly bound projectile electrons may be excited or ionized as a result of the interaction with the target, and they themselves may excite or ionize the electrons of the target atom; i.e., they participate actively in the collision.

Theoretical and experimental investigations of the screening effect in ion-atom collisions have led to some surprising results [2-5]. At first glance, one would expect that the cross sections of the collision processes, as compared to the case of bare ion projectiles, are smaller for the impact of partially stripped ions due to the weaker screened Coulomb potential. However, it turned out that this is not always the case: under certain collision conditions the cross sections increase as a result of the presence of the electrons in the projectile. One of the reasons for this "anti-screening" effect can be understood by considering the shape of the screened Coulomb potential, which at some interaction distances may change more rapidly than that of the pure Coulomb potential. Particularly at low collision velocities, the excitation or ionization amplitudes are more sensitive to the sharper changes of the potential than to the magnitude of the potential itself, resulting in enhanced cross sections [3]. As an example, we mention one of the most striking manifestations of the antiscreening effect that was observed in collisions of 1 and $1.5 \mathrm{MeV} \mathrm{amu}^{-1}$ fluorine ions with helium and hydrogen targets [4]. In this experiment it was found, surprisingly, that the yield of the so-called binary-encounter peak in the spectrum of the forward-ejected electrons increased when the charge state of the projectile decreased from 9 (fully stripped ions) to 3.

As mentioned above, at high impact energies the projectile electrons may play an active role in the collision. In this case the dynamics of the projectile electrons can be well understood by the concept of the "quasifree electron scattering" which regards the electrons as if they were free particles that interact with the target independently from the projectile nucleus. In the quantitative treatment of the quasifree electron scattering one has to take into account the fact that the velocity of the electrons is not sharply defined but is broadened due their momentum distribution (Compton profile) in the projectile ion. As an example of the applications of this model we mention its successful use for the description of the resonant transfer 
and excitation (RTE) process [6]. RTE was first observed in collisions of highly charged ions with a light atom [7], i.e., in an inverse scattering system from the point of view of the quasifree electron. It is a correlated two-electron process involving the transfer of a target electron to the projectile with the simultaneous excitation of a projectile electron, giving rise to doubly excited states. RTE is analogous to the dielectronic recombination (DR) occurring in electron-atom collisions (the inverse process of the Auger decay); that is why the quasifree electron model is well suited for its description. As a further example, we mention the recent experimental investigations of the scattering of positronium (Ps) on atoms [8,9]. The obtained results suggest that the electron constituent of Ps plays the dominant role in the collision, and it interacts with the target as a quasifree particle: The measured total cross sections revealed an unexpected, strong similarity with those for equivelocity electron projectiles.

Historically, the term antiscreening was introduced to characterize only the active participation (excitation or ionization) of the projectile electrons in the target ionizing process $[10,11]$. Here we use it in a broader sense: We mean by antiscreening all kind of effects that result in an enhancement of the cross section due to the presence of the electrons in the projectile ion (including their passive participation in the target ionization; see above).

In this paper we report on the experimental investigation of the multiple ionization of $\mathrm{He}, \mathrm{Ne}, \mathrm{Ar}$, and $\mathrm{Kr}$ by the simplest neutral atomic projectile, $\mathrm{H}^{0}$. The energy range of the measurements was $75-300 \mathrm{keV}$. Considering the role of the projectile electron, this is an intermediate range. From one side the static screening effect may influence significantly the process. From the other side the collision velocity is high enough to view the electron as a quasifree particle, i.e., excitation or ionization of the target by the projectile electron cannot be excluded. (The threshold proton energies of the equivelocity electron-impact ionization of the outermost shells of the targets are as follows: He, $45.2 \mathrm{keV}$; $\mathrm{Ne}$, $39.7 \mathrm{keV}$; Ar, $28.9 \mathrm{keV}$; Kr, $25.9 \mathrm{keV}$.) The $\mathrm{H}^{0}$ projectile is particularly interesting from a theoretical point of view, considering that at large collision velocities the first-order ionization theories predict equal cross sections for the proton and electron constituents. A recent theoretical analysis carried out for the excitation of highly charged hydrogen-like ions in collisions with equivelocity electrons and protons [12] showed that electronic projectiles are not less effective in inducing the excitation than the protons, even at collision velocities slightly above the threshold for the electron-impact excitation. This finding indicates a non-negligible contribution of the electron constituent in the collisions studied in the present work. However, a question remains regarding how far the basic assumption of quasifree electron scattering model, namely that the contributions of the electron and proton constituents can be added incoherently, is valid.

Besides the theoretical interest, we mention here a specialty of the $\mathrm{H}^{0}$ projectile, namely its role played in the field of the fusion research [13]. Injection of energetic neutral hydrogen beams into the fusion plasma is a routinely used method to heat the plasma. For the optimization of the heating, accurate knowledge of the basic collision processes of $\mathrm{H}^{0}$ with the constituents of the plasma (electrons, protons, impurity ions) is necessary. Neutral hydrogen beams are also used for diagnostic purposes: Information about the characteristics of the plasma can be obtained by spectroscopy of the photons emitted by either the excited $\mathrm{H}^{0}$ atoms of the injected beam or by the excited atoms (ions) of the plasma produced by charge exchange in collisions with the injected $\mathrm{H}^{0}$ atoms.

In spite of the large theoretical and practical importance of the collisions involving atomic hydrogen projectiles, there have been very few experimental investigations of the multiple ionization of atoms by $\mathrm{H}^{0}$ impact. The first measurement, where the final charge states of both collision partners were observed, was reported by Afrosimov et al. [14]. In this experiment carried out for $\mathrm{H}^{0}$ on Xe collisions in the energy range 5-50 keV the authors determined cross sections for recoil target charges ranging from 1 to 4 , and for projectile charges $+1,0$, and -1 . Further experiments in which the cross sections for the simultaneous ionization of both collision partners were measured: $\mathrm{H}^{0}+\mathrm{He}, \mathrm{Xe}(50-1200 \mathrm{keV})[15]$; $\mathrm{H}^{0}+\mathrm{He}, \mathrm{Ar}(0.5-2 \mathrm{MeV})[16] ; \mathrm{H}^{0}+\mathrm{He}(25-1000 \mathrm{keV})$ $[17,18]$.

Our present work is primarily of experimental character in the sense that information about the role of the electron in $\mathrm{H}^{0}$ was obtained by repeating the measurements with protons under the same experimental conditions. In our theoretical calculations carried out in the framework of the classical trajectory Monte Carlo (CTMC) method we did not attempt to describe the active role of the projectile electron; its effect in the collision processes was taken into account only as a simple static screening.

The paper is organized as follows. In Sec. II we present the applied experimental setup and method. In Sec. III we discuss the description of the collisional multiple ionization of atoms in the framework of the independent particle model. In this section we briefly outline also the CTMC method used for the calculation of the single-electron ionization and capture probabilities, the basic quantities that are needed to determine the multiple ionization cross sections. In Sec. IV we compare our experimental data with the theoretical ones for both projectiles, as well as we investigate the effect of the electron in $\mathrm{H}^{0}$ by analyzing the ratios of the cross sections belonging to bare and screened projectile impact. Finally, the conclusions are drawn in Sec. V. Atomic units are used throughout the paper, unless noted otherwise.

\section{EXPERIMENTAL SETUP AND METHOD}

The measurements were made at the 1.5 MV Van de Graaff accelerator of Atomki. The scheme of the experimental setup is shown in Fig. 1 for the case when the projectile was $\mathrm{H}^{0}$. The neutral beam was produced from the $\mathrm{H}^{+}$beam of the accelerator via charge exchange with the residual gas of the beam channel. An electrostatic deflector was used to remove the charged components of the beam (not shown in the figure). A collimator with a length of $50 \mathrm{~cm}$ defines the final $\mathrm{H}^{0}$ beam of $0.5 \mathrm{~mm}$ diameter. The remaining charged ions produced in the collimator are swept away by an electrostatic beam cleaner mounted just in front of an effusive gas target. For the measurements with proton projectiles the arrangement in Fig. 1 differed only in that we used a four-component electrostatic charge-state selector (see, e.g., Ref. [19]) for cleaning the 


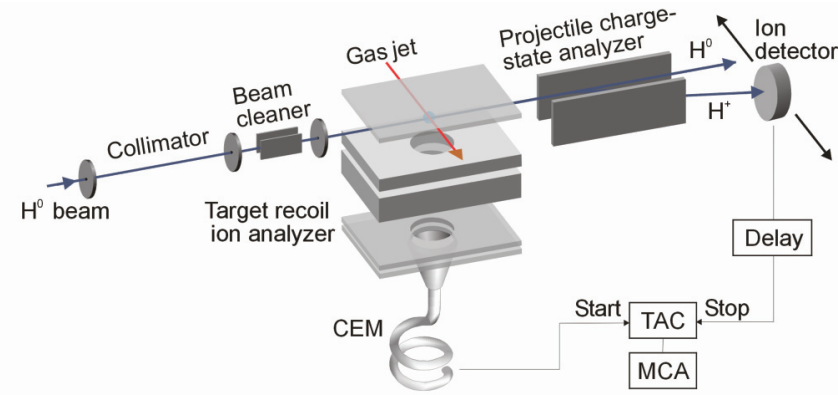

FIG. 1. (Color online) Scheme of the experimental setup. For the sake of the better visibility the recoil target ion analyzer is strongly enlarged compared to the other components of the setup.

beam. The $\mathrm{H}^{+}\left(\mathrm{H}^{0}\right)$ contamination of the $\mathrm{H}^{0}\left(\mathrm{H}^{+}\right)$beam was on the order of $0.1 \%$.

The ions produced by the interaction of the beam with the target are extracted from the collision region by a weak electric field $(40 \mathrm{~V} / \mathrm{cm})$ and enter into a recoil target ion analyzer built for the purpose of the present investigations. In the analyzer the ions of charge state $q$ are accelerated to energy $q U$ where $U$ is the accelerating voltage $(U=3.1 \mathrm{kV}$ was used in the measurements). The electrode system of the analyzer (see Fig. 2) was designed to collect the ions with an efficiency of almost $100 \%$. For optimization we used the SIMION 3D ion optics computer program (Version 8.0). The accelerated ions are detected by a ceramic channel electron multiplier (CEM).

The scattered outgoing projectile ions are charged-state analyzed by means of an electrostatic deflector and detected by a fast ion detector [20]. The delayed pulse from this detector provides the stop signal for the time-of-flight measurement of the recoil ions. The time difference between the pulse from the recoil ion detector and the delayed pulse from the scattered ion detector is measured by a time-to-amplitude converter (TAC). Time spectra were obtained by pulse-height analysis of the TAC output using a multichannel amplitude analyzer (MCA). A typical time spectrum is shown in Fig. 3.

The pressure in the measuring chamber without and with the target gas was $10^{-6}$ and $3 \times 10^{-5} \mathrm{mbar}$, respectively. The density of the target gas was low enough for the fulfillment of

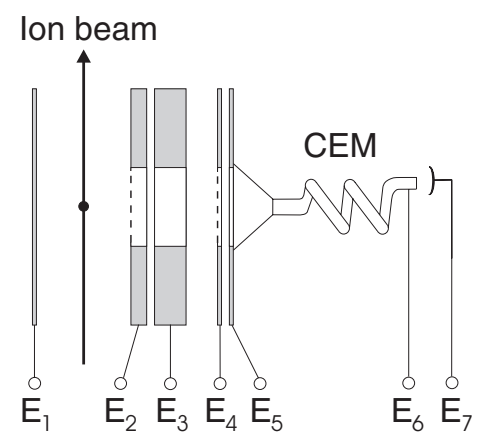

FIG. 2. The electrode system of the recoil target ion analyzer: $E_{1}$, "push" electrode $(20 \mathrm{~V}) ; E_{2}$, "pull" electrode with grid $(-20 \mathrm{~V})$; $E_{3}$, focusing electrode $(-150 \mathrm{~V}) ; E_{4}$, accelerating electrode with grid $(-3.1 \mathrm{kV}) ; E_{5}$, funnel of CEM $(-2.9 \mathrm{kV}) ; E_{6}$, end of CEM $(-0.2 \mathrm{kV}) ; E_{7}$, collector electrode of CEM $(0 \mathrm{~V})$.

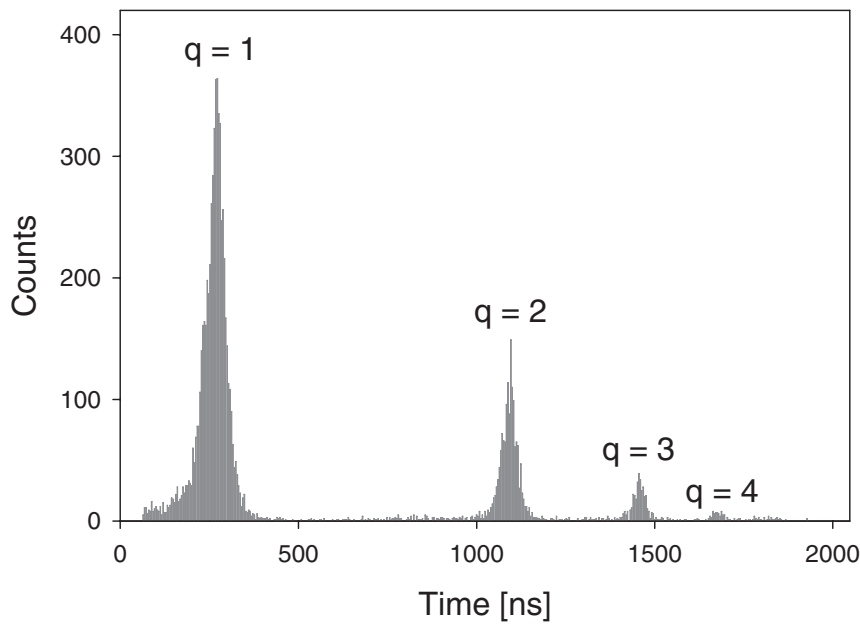

FIG. 3. Time spectrum of the recoil target ions measured for $300 \mathrm{keV} \mathrm{H}^{0}$ on $\mathrm{Kr}$ collisions in coincidence with the outgoing $\mathrm{H}^{+}$ projectiles.

the condition of the single collision. The contribution of the residual gas to the yield of the recoil target ions was less than $0.1 \%$.

We took time spectra for target gases $\mathrm{He}, \mathrm{Ne}, \mathrm{Ar}$, and $\mathrm{Kr}$, for both proton and $\mathrm{H}^{0}$ impact, at five projectile energies (75, $100,150,200$, and $300 \mathrm{keV})$. The coincidence runs were made for charge states 0 and +1 of the scattered projectiles. We did not measure the reactions leading to charge state -1 , because the coincidence yields were very small in these cases. As a consistency check, we always took also a spectrum without charge-state selection, i.e., we measured directly the sum of the yields of the different reaction channels. The measuring time of the spectra varied between 200-2000 s. For example, the measurement of the spectrum displayed in Fig. 3 took about five minutes. The corresponding count rates (scattered ions: $400 \mathrm{~s}^{-1}$; recoil ions: $100 \mathrm{~s}^{-1}$; rate of the coincidence events: $20 \mathrm{~s}^{-1}$ ) are typical data that characterize the present coincidence experiment.

From the coincidence yields we determined only relative cross sections. Absolute cross sections $\sigma_{q}^{i j}$ ( $i$ and $j$ are the initial and final charge of the projectile, respectively; $q$ is the charge of the recoil target ion) were obtained by normalizing our data to the absolute measurements of DuBois and Kövér [18] carried out for $\mathrm{H}^{0}$ on He collisions. The normalization was made at the measuring point of $300 \mathrm{keV}$ impact energy for the total ionization cross section $\sum_{j, q} \sigma_{q}^{0 j}(j=0,1$ and $q=1,2)$.

\section{THEORY}

\section{A. Three-body CTMC approach}

For the theoretical treatment of the multiple vacancy creation we assumed the validity of the independent particle model (IPM). In IPM the description of the many-electron collision system is reduced to the problem of a three-body system consisting of the projectile, an active electron, and the target ion core. For the solution of the three-body problem we applied the classical trajectory Monte Carlo method (CTMC). The method is based on the numerical solution of the classical 
equations of motion for a large number of trajectories of the interacting particles under randomly chosen initial conditions $[21,22]$.

The details of the CTMC computer code are given in Ref. [23]. Briefly, it solves Newton's nonrelativistic equations of motion for the three particles. The randomly selected initial conditions are the impact parameter and five further parameters defining the position and velocity vector of the target electron moving in classical orbits. The ranges of the latter parameters are constrained to give the binding energy of the electron in the considered shell of the atom. The role of the passive electrons of the target core is taken into account by an effective potential. For this purpose in the present work a model potential developed by Green et al. [24] was used, which has the following form:

$$
V(r)=-\{Z-(N-1)[1-\Omega(r)]\} / r,
$$

where $Z$ is the nuclear charge, $N$ is the number of the electrons in the atom, and

$$
\Omega(r)=\{(\eta / \xi)[\exp (\xi r)-1]+1\}^{-1} .
$$

The values of $\eta$ and $\xi$ were taken from Garvey et al. [25].

For the generation of the initial values of the position and velocity coordinates of the electron from a set of uniformly distributed variables we applied the general procedure suggested by Reinhold and Falcón [26] for non-Coulombic systems.

For the calculation of the cross section of a multielectron process in the framework of IPM the most straightforward way is the impact-parameter formulation of the three-body scattering problem. From the solution of the problem for a specific shell one obtains single-electron probabilities as functions of the impact parameter $b$ for the basic collision processes: excitation, ionization, and electron capture. Denoting these probabilities by $p_{e}(b), p_{i}(b)$, and $p_{c}(b)$, the relation $p_{e}(b)+p_{i}(b)+p_{c}(b)=1$ holds.

The treatment of the multiple vacancy production for proton and $\mathrm{H}^{0}$ projectile is different; we discuss the two cases separately.

\section{Proton impact}

Let us consider a shell with $N$ electrons. The probability that in the collision $n$ electrons are ejected (ionized) and $m$ electrons are captured is expressed by the following binomial expression:

$$
P_{i^{n} c^{m}}=\left(\begin{array}{c}
N \\
n
\end{array}\right)\left(\begin{array}{c}
N-n \\
m
\end{array}\right) p_{i}^{n} p_{c}^{m} p_{e}^{N-(n+m)} .
$$

For an atom having $Q$ shells the probability of the multiple vacancy creation is a product of the probabilities of the individual shells given by Eq. (2). We introduce the following notations. For the $k$ th shell $(k=1, \ldots, Q)$ the number of the electrons is $N_{k}$; the single electron excitation, ionization, and capture probabilities are $p_{e k}, p_{i k}$, and $p_{c k}$, respectively; the number of the electrons ejected from the shell is $n_{k}$; the number of the electrons captured from the shell is $m_{k}$. The probability of the creation of the vacancy configuration $\left(n_{1}, n_{2}, \ldots, n_{Q}\right.$, $\left.m_{1}, m_{2}, \ldots, m_{Q}\right)$ is given by

$$
\begin{aligned}
& P_{i^{n_{1}, n_{2}, \ldots, n} Q} Q c^{m_{1}, m_{2}, \ldots, m} Q \\
& \quad=\prod_{k=1}^{Q}\left(\begin{array}{c}
N_{k} \\
n_{k}
\end{array}\right)\left(\begin{array}{c}
N_{k}-n_{k} \\
m_{k}
\end{array}\right) p_{i k}^{n_{k}} p_{c k}^{m_{k}} p_{e k}^{N_{k}-\left(n_{k}+m_{k}\right)} .
\end{aligned}
$$

The recoil charge state of the atom belonging to the considered specific configuration is $q=\sum_{k=1}^{Q}\left(n_{k}+m_{k}\right)$. We introduce the short notation $\gamma$ for the configuration $\left(n_{1}, n_{2}, \ldots, n_{Q}\right.$, $\left.m_{1}, m_{2}, \ldots, m_{Q}\right)$, and $P_{\gamma}(q)$ for its probability expressed by Eq. (3). For the determination of the probability of the recoil charge state $j$ created in the collision we have to sum over all possible configuration with the condition $q=j$ :

$$
P_{j}=\sum_{\gamma} P_{\gamma}(q) \delta_{q j}
$$

In addition to the direct collisional multiple vacancy creation, relaxation processes following the ionization of the inner shells may also contribute to the experimentally observed recoil charge state. The electron emission due the Auger, Coster-Krönig, and shake-off processes and subsequent possible Auger cascades may result in additional vacancies in the outer shells. To a good approximation, the relaxation processes are well separated in time from the direct vacancy creation, therefore their effects can be treated independently from the considered collisional event.

For the inclusion of the relaxation effects we followed the procedure suggested by Montanari et al. [27]. Since the Auger emission depends on the considered shell, we have to write the probability of the vacancy creation separately for each shell (subshell). To characterize the Auger process, for a certain $\mu$ subshell we define the branching ratio $F_{\mu, k}$ as the probability that a single vacancy creation in the considered subshell is followed by Auger electron emission that results in additional $k$ vacancies in the outer shells (i.e., the total number of the vacancies in the final state is $k+1)$.

Let us denote the number of the vacancies in the $\mu$ subshell created primarily in the collision by $q_{\mu}$, i.e., $q_{\mu}=n_{\mu}+m_{\mu}$. The probability of the production of $q_{\mu}$ primary vacancies in the $\mu$ subshell is as follows:

$$
P_{\mu}\left(q_{\mu}\right)=\left(\begin{array}{c}
N_{\mu} \\
n_{\mu}
\end{array}\right)\left(\begin{array}{c}
N_{\mu}-n_{\mu} \\
m_{\mu}
\end{array}\right) p_{i}^{n_{\mu}} p_{c}^{m_{\mu}} p_{e}^{N_{\mu}-\left(n_{\mu}+m_{\mu}\right)} .
$$

As a result of the Auger process, $P_{\mu}\left(q_{\mu}\right)$ changes. Let us denote the modified probability by $\mathcal{P}_{\mu}\left(\alpha_{\mu}\right)$ where $\alpha_{\mu}=q_{\mu}+k$. Montanari et al. [27] have derived the following expressions for the single, double, and triple ionization probabilities including the Auger transitions:

$$
\begin{aligned}
& \mathcal{P}_{\mu}(1)=P_{\mu}(1) F_{\mu, 0}, \\
& \mathcal{P}_{\mu}(2)=P_{\mu}(1) F_{\mu, 1}+P_{\mu}(2) F_{\mu, 0}^{2}, \\
& \mathcal{P}_{\mu}(3)=P_{\mu}(1) F_{\mu, 2}+2 P_{\mu}(2) F_{\mu, 0} F_{\mu, 1}+P_{\mu}(3) F_{\mu, 0}^{3} .
\end{aligned}
$$

For the whole atom the probability of charge state $j$ in the final state is

$$
P_{j}=\sum_{\alpha_{1}+\alpha_{2}+\cdots+\alpha_{Q}=j} \prod_{\mu=1}^{Q} \mathcal{P}_{\mu}\left(\alpha_{\mu}\right)
$$


The corresponding cross section is

$$
\sigma_{j}=2 \pi \int_{0}^{\infty} b P_{j}(b) d b .
$$

In the absence of Auger processes Eqs. (5)-(7) are expected to be equivalent with Eqs. (3) and (4). However, this is not so, because the product in Eq. (7) does not contain factors that account for events when the electrons are only excited in the shell. Therefore, in our procedure we extended the expressions (7) given by Montanari et al. [27] in the following way:

$$
\begin{aligned}
& \mathcal{P}_{\mu}(0)=P_{\mu}(0) \\
& \mathcal{P}_{\mu}(1)=P_{\mu}(1) F_{\mu, 0} \\
& \mathcal{P}_{\mu}(2)=P_{\mu}(1) F_{\mu, 1}+P_{\mu}(2) F_{\mu, 0}^{2}, \\
& \mathcal{P}_{\mu}(3)=P_{\mu}(1) F_{\mu, 2}+2 P_{\mu}(2) F_{\mu, 0} F_{\mu, 1}+P_{\mu}(3) F_{\mu, 0}^{3} .
\end{aligned}
$$

\section{H $^{0}$ impact}

For the description of the multiple vacancy creation we applied the same procedure as for proton impact, but the presence of the extra electron in the projectile required the following considerations:

(i) For the treatment of the ionization of the target atom the three-body problem was solved by applying a completely screened Coulomb potential for the projectile.

(ii) The ionization of the projectile atom ("electron loss", EL) was included by solving the inverse scattering problem, i.e., ionization of $\mathrm{H}^{0}$ by impact of the target atom. In this approach the target atom was considered as a pointlike particle with completely screened Coulomb potential.

(iii) The contribution of the electron capture into the bound states of both the projectile and the target atom turned out to be very small, therefore it was neglected.

Introducing the notation $P_{\mathrm{EL}}$ for the probability of the electron loss, for the reaction channel with $\mathrm{H}^{0}$ outgoing projectile in the final state Eq. (7) is modified as

$$
P_{j}^{0}=\left(1-P_{\mathrm{EL}}\right) \sum_{\alpha_{1}+\alpha_{2}+\cdots+\alpha_{Q}=j} \prod_{\mu=1}^{Q} \mathcal{P}_{\mu}\left(\alpha_{\mu}\right) .
$$

For $\mathrm{H}^{+}$outgoing projectile,

$$
P_{j}^{0}=P_{\mathrm{EL}} \sum_{\alpha_{1}+\alpha_{2}+\cdots+\alpha_{Q}=j} \prod_{\mu=1}^{Q} \mathcal{P}_{\mu}\left(\alpha_{\mu}\right) .
$$

In the above expressions $\mathcal{P}_{\mu}\left(\alpha_{\mu}\right)$ are evaluated in the same way as for proton impact, but the $P_{\mu}\left(q_{\mu}\right)$ probabilities in Eq. (5) are calculated by considering only ionization:

$$
P_{\mu}\left(q_{\mu}\right)=\left(\begin{array}{c}
N_{\mu} \\
n_{\mu}
\end{array}\right) p_{i}^{n_{\mu}} p_{e}^{N_{\mu}-n_{\mu}}
$$

\section{B. Simplified nCTMC model}

The assumption of the three-body CTMC model that the ionization of all the electrons in a given subshell can be treated energetically on equal footing is very questionable. As an example, let us consider the double ionization of an atom: Obviously the removal of the second electron is energetically less favorable than that of the first electron. For a more realistic description of the multiple ionization Olson et al. [28] developed the so-called nCTMC model. In this model the classical equations of motion are solved for the full many-body collision system consisting of the $N$ electrons and the nucleus of the target atom, as well as the projectile ion. The method includes all the forces between the particles, except the electron-electron interactions. The latter ones are partly taken into account by effective potentials describing the interaction of each electrons with the target nucleus separately.

The nCTMC method does not consider strictly the shell structure of the atom: The binding energies of the electrons are the sequential 1 st, $2 \mathrm{nd}, \ldots, N$ th ionization energies. This ensures that the total electronic energy of the multiply ionized atom is correct. The solution of the many-body equations determines the trajectories of all the electrons simultaneously, and thereby the cross sections for the multiple ionization and capture processes can be directly determined, i.e., one does not need to carry out further statistical analysis.

A disadvantage of $\mathrm{nCTMC}$ is that the necessary numerical calculations are very time-consuming: One has to solve $6(N+$ 2) coupled, first-order differential equations for a large number $\left(\sim 10^{5}\right)$ of collisions. This was one of the reasons that in the present work we used a simplified version of the model. The assumptions of our model are as follows:

(i) We assume that IPM still can be applied for the treatment of the many-body problem outlined above. Since in nCTMC the electron-electron interactions are neglected, the only couplings between the motions of the electrons are due to the subsequent scatterings of the projectile on the electrons. However, because of the large mass difference between the electron and the projectile ion, such scatterings result in a very small change in the projectile trajectory. This means that each electron feels the same time-dependent perturbation potential of the projectile, therefore their motions are independent of each other. Mathematically, the equations of motion for the individual electrons decouple, and the many-body problem reduces to a three-body problem. As a result of this approximation, we could use our three-body CTMC code in the calculations. We note that, although in this way the use of the name "nCTMC" is not justified, we still keep it because it reflects better the many-body character of the vacancy creation than CTMC.

(ii) We made our nCTMC calculations only for the electrons of the outermost shell of the target atoms, considering that in the energy range of the present work the outermost shell has the dominant contribution to the multiple vacancy creation cross sections. This means that we considered the first and second electron removals from $\mathrm{He}$, and the first, second, ..., sixth electron removals from the outermost shell of the heavier target atoms (Ne: $L_{23}$; Ar: $\left.M_{23} ; \mathrm{Kr}: N_{23}\right)$.

In our nCTMC model the inner shells of the atom were regarded as a core whose contribution to the probability of a given vacancy configuration was determined by the traditional CTMC method as discussed in the previous section. Since for the outermost shell we solve also the three-body equations, the probabilities of the various vacancy configurations are not obtained automatically as in the original nCTMC model, but still one needs statistical considerations to derive them. In the following we use the notations $p_{i 1}^{\prime}, p_{i 2}^{\prime}, \ldots, p_{i N_{1}}^{\prime}$, $p_{c 1}^{\prime}, p_{c 2}^{\prime}, \ldots, p_{c N_{1}}^{\prime}$, and $p_{e 1}^{\prime}, p_{e 2}^{\prime}, \ldots, p_{e N_{1}}^{\prime}$ for the single electron 
ionization, capture, and excitation probabilities that are obtained in our simplified nCTMC model by taking the sequential 1 st, $2 \mathrm{nd}, \ldots, N_{1}$ th ionization energies of the target atom as the binding energy of the electron $\left(N_{1}\right.$ equals 2 and 6 for $\mathrm{He}$ and the heavier targets, respectively). As an example, let us consider the double ionization taking place in the outermost shell of a heavy atom. In our model the probability of the process is

$$
P=\left(\begin{array}{c}
N_{1} \\
2
\end{array}\right) p_{i 1}^{\prime} p_{i 2}^{\prime} p_{e 3}^{\prime} p_{e 4}^{\prime} \cdots p_{e N_{1}}^{\prime} P(\text { core }),
$$

while the corresponding expression in CTMC is

$$
P=\left(\begin{array}{c}
N_{1} \\
2
\end{array}\right) p_{i 1}^{2} p_{e 1}^{\left(N_{1}-2\right)} P(\text { core })
$$

Here $P$ (core) accounts for the contribution of the inner shells. Notice that for $p_{i k}^{\prime}=p_{i 1}\left(k=1, \ldots, N_{1}\right)$ Eq. (13) is identical with Eq. (14).

Since in nCTMC the electrons in the same subshell are not equivalent, one has to take into account the time order of the regarded process. As an example, let us consider the simultaneous ionization and capture of two electrons in the outer shell. Two kind of time orders are possible; the corresponding probabilities are

$$
P^{(1)}=\left(\begin{array}{c}
N_{1} \\
1
\end{array}\right)\left(\begin{array}{c}
N_{1}-1 \\
1
\end{array}\right) p_{i 1}^{\prime} p_{c 2}^{\prime} p_{e 3}^{\prime} p_{e 4}^{\prime} \cdots p_{e N_{1}}^{\prime} P(\text { core })
$$

(ionization followed by capture), and

$$
P^{(2)}=\left(\begin{array}{c}
N_{1} \\
1
\end{array}\right)\left(\begin{array}{c}
N_{1}-1 \\
1
\end{array}\right) p_{i 2}^{\prime} p_{c 1}^{\prime} p_{e 3}^{\prime} p_{e 4}^{\prime} \cdots p_{e N_{1}}^{\prime} P(\text { core })
$$

(capture followed by ionization). We symmetrize the probabilities by taking the average of $P^{(1)}$ and $P^{(2)}$.

In a similar way, the time order of the different processes was taken into account in deriving the probabilities of higher ionization degrees with and without assuming single electron capture. With increasing multiplicity the number of the possible time orders rapidly increases, resulting in complicated expressions. The situation becomes even more complex when the vacancy creation in the core simultaneous with that in the outermost shell is also included. In our nCTMC model we considered all the cases that contribute to recoil charge states $q=1,2$, and 3 for both the pure ionization and the electron capture channel. As far as the Auger processes are concerned, their effect was included in the same way as for CTMC.

\section{RESULTS AND DISCUSSION}

The cross sections $\sigma_{q}^{i j}$ measured in the present work are listed in Tables I-III and presented graphically in Figs. 4-7. Since we used the He target only for normalization purposes, we did not measure the very small double ionization cross section values in this case. However, for the sake of completeness we plotted in Fig. 4 double ionization cross sections taken from the literature, namely $\sigma_{2}^{11}$ and $\sigma_{2}^{10}$ measured by Shah and Gilbody [29], and $\sigma_{2}^{00}$ and $\sigma_{2}^{01}$ measured by DuBois and Kövér [18].

We note that our measured data for proton impact agree quite well with results of previous experiments (see, for

\begin{tabular}{|c|c|c|c|c|}
\hline$E(\mathrm{keV})$ & $\sigma_{1}^{11}$ & $\sigma_{1}^{10}$ & $\sigma_{1}^{00}$ & $\sigma_{1}^{01}$ \\
\hline \multicolumn{5}{|c|}{$\mathrm{He}$} \\
\hline 75 & $89.4 \pm 17.7$ & $85.9 \pm 17.0$ & $50.7 \pm 10.6$ & $22.8 \pm 4.49$ \\
\hline 100 & $92.3 \pm 18.2$ & $47.2 \pm 9.33$ & $44.2 \pm 8.71$ & $20.1 \pm 3.96$ \\
\hline 150 & $82.6 \pm 16.3$ & $16.5 \pm 3.30$ & $40.4 \pm 7.94$ & $23.5 \pm 4.63$ \\
\hline 200 & $71.4 \pm 14.1$ & $6.03 \pm 1.22$ & $31.9 \pm 6.28$ & $14.2 \pm 2.8$ \\
\hline 300 & $55.1 \pm 10.9$ & $\begin{array}{c}1.46 \pm 0.32 \\
\mathrm{Ne}\end{array}$ & $23.1 \pm 4.55$ & $12.2 \pm 2.40$ \\
\hline 75 & $152 \pm 29.9$ & $119 \pm 21.2$ & $56.9 \pm 11.2$ & $50.9 \pm 10.0$ \\
\hline 100 & $152 \pm 30.1$ & $88.5 \pm 17.5$ & $56.1 \pm 11.1$ & $60.3 \pm 11.9$ \\
\hline 150 & $137 \pm 27.1$ & $34.0 \pm 6.22$ & $46.2 \pm 9.10$ & $58.1 \pm 11.4$ \\
\hline 200 & $124 \pm 24.6$ & $21.5 \pm 4.26$ & $40.2 \pm 7.92$ & $55.0 \pm 10.8$ \\
\hline 300 & $109 \pm 21.5$ & $\begin{array}{c}6.05 \pm 1.13 \\
\mathrm{Ar}\end{array}$ & $35.3 \pm 6.95$ & $26.8 \pm 5.28$ \\
\hline 75 & $472 \pm 93.1$ & $199 \pm 39.3$ & $199 \pm 39.4$ & $301 \pm 59.5$ \\
\hline 100 & $465 \pm 91.8$ & $96.2 \pm 19.0$ & $164 \pm 32.3$ & $290 \pm 57.2$ \\
\hline 150 & $429 \pm 84.6$ & $31.0 \pm 6.14$ & $88.7 \pm 17.5$ & $205 \pm 40.5$ \\
\hline 200 & $377 \pm 74.3$ & $9.58 \pm 1.92$ & $66.1 \pm 13.0$ & $131 \pm 25.9$ \\
\hline 300 & $325 \pm 64.2$ & $\begin{array}{c}1.86 \pm 0.40 \\
\mathrm{Kr}\end{array}$ & $43.4 \pm 8.60$ & $85.3 \pm 16.9$ \\
\hline 75 & $591 \pm 117$ & $221 \pm 43.7$ & $127 \pm 25.1$ & $103 \pm 20.4$ \\
\hline 100 & $569 \pm 112$ & $175 \pm 34.6$ & $130 \pm 25.7$ & $155 \pm 30.5$ \\
\hline 150 & $499 \pm 98.5$ & $32.1 \pm 6.35$ & $98.6 \pm 19.5$ & $149 \pm 29.4$ \\
\hline 200 & $474 \pm 93.6$ & $9.71 \pm 1.92$ & $92.1 \pm 18.2$ & $143 \pm 28.2$ \\
\hline 300 & $412 \pm 81.4$ & $1.12 \pm 0.25$ & $84.5 \pm 16.7$ & $181 \pm 35.8$ \\
\hline
\end{tabular}
example, the paper of DuBois and Manson [30] presenting
TABLE I. Cross sections for single ionization of $\mathrm{He}, \mathrm{Ne}, \mathrm{Ar}$, and $\mathrm{Kr}$ by proton and $\mathrm{H}^{0}$ impact in units of $10^{-18} \mathrm{~cm}^{2}$.

a large amount of cross section data for the multiple ionization of noble gases which were obtained from the literature and supplemented also with some additional measurements by the authors).

Although in the time spectra for Ne target taken at some impact energies we observed the peak belonging to target recoil charge $q=3$, with an acceptable experimental uncertainty

TABLE II. Cross sections for double ionization of $\mathrm{Ne}, \mathrm{Ar}$, and $\mathrm{Kr}$ by proton and $\mathrm{H}^{0}$ impact in units of $10^{-18} \mathrm{~cm}^{2}$.

\begin{tabular}{lcccc}
\hline \hline$E(\mathrm{keV})$ & $\sigma_{2}^{11}$ & $\sigma_{2}^{10}$ & $\sigma_{2}^{00}$ & $\sigma_{2}^{01}$ \\
\hline \multicolumn{5}{c}{$\mathrm{Ne}$} \\
75 & $7.24 \pm 1.46$ & $13.4 \pm 2.70$ & $4.09 \pm 0.92$ & $2.97 \pm 0.60$ \\
100 & $7.82 \pm 1.61$ & $11.0 \pm 2.18$ & $4.10 \pm 0.83$ & $4.37 \pm 0.88$ \\
150 & $10.4 \pm 2.07$ & $5.68 \pm 1.18$ & $3.71 \pm 0.78$ & $5.17 \pm 1.04$ \\
200 & $10.3 \pm 2.06$ & $3.71 \pm 0.74$ & $3.44 \pm 0.77$ & $5.83 \pm 1.18$ \\
300 & $6.49 \pm 1.31$ & $1.05 \pm 0.22$ & $2.50 \pm 0.55$ & $3.06 \pm 0.62$ \\
& \multicolumn{4}{c}{} \\
75 & $71.7 \pm 14.5$ & $57.9 \pm 11.7$ & $36.4 \pm 7.54$ & $52.4 \pm 10.6$ \\
100 & $66.5 \pm 13.8$ & $26.1 \pm 5.34$ & $23.4 \pm 4.82$ & $53.0 \pm 10.6$ \\
150 & $58.3 \pm 12.0$ & $8.69 \pm 1.77$ & $11.0 \pm 2.30$ & $45.4 \pm 9.03$ \\
200 & $39.6 \pm 8.02$ & $2.56 \pm 0.58$ & $6.61 \pm 1.35$ & $27.7 \pm 5.53$ \\
300 & $25.6 \pm 5.25$ & $0.69 \pm 0.17$ & $4.99 \pm 1.03$ & $19.9 \pm 3.97$ \\
& & $\mathrm{Kr}$ & & \\
75 & $114 \pm 23.3$ & $114 \pm 22.8$ & $21.4 \pm 4.52$ & $18.2 \pm 3.74$ \\
100 & $84.2 \pm 16.9$ & $81.0 \pm 16.4$ & $22.3 \pm 4.47$ & $31.4 \pm 6.24$ \\
150 & $63.4 \pm 12.9$ & $13.3 \pm 2.67$ & $16.6 \pm 3.39$ & $37.8 \pm 7.53$ \\
200 & $62.1 \pm 13.0$ & $4.21 \pm 0.87$ & $14.5 \pm 3.14$ & $37.3 \pm 7.44$ \\
300 & $40.0 \pm 8.09$ & $1.43 \pm 0.36$ & $10.9 \pm 2.24$ & $47.2 \pm 9.38$ \\
\hline \hline
\end{tabular}


TABLE III. Cross sections for triple ionization of $\mathrm{Ar}$ and $\mathrm{Kr}$ by proton and $\mathrm{H}^{0}$ impact in units of $10^{-18} \mathrm{~cm}^{2}$.

\begin{tabular}{lcccc}
\hline \hline$E(\mathrm{keV})$ & $\sigma_{3}^{11}$ & $\sigma_{3}^{10}$ & $\sigma_{3}^{00}$ & $\sigma_{3}^{01}$ \\
\hline \multicolumn{5}{c}{$\mathrm{Ar}$} \\
75 & $5.14 \pm 1.90$ & $3.52 \pm 0.97$ & $2.84 \pm 0.99$ & $2.20 \pm 0.57$ \\
100 & $6.04 \pm 2.99$ & $0.76 \pm 0.33$ & $1.75 \pm 0.61$ & $2.81 \pm 0.62$ \\
150 & $4.20 \pm 1.83$ & $0.39 \pm 0.13$ & $1.45 \pm 0.65$ & $2.38 \pm 0.55$ \\
200 & $2.34 \pm 0.70$ & $0.18 \pm 0.10$ & $0.67 \pm 0.20$ & $1.88 \pm 0.40$ \\
300 & $1.76 \pm 0.68$ & $0.21 \pm 0.06$ & $0.74 \pm 0.21$ & $1.49 \pm 0.32$ \\
& & $\mathrm{Kr}$ & & \\
75 & $13.2 \pm 4.09$ & $10.7 \pm 2.38$ & $3.19 \pm 0.74$ & $1.70 \pm 0.39$ \\
100 & $8.87 \pm 2.69$ & $9.40 \pm 2.17$ & $3.01 \pm 0.71$ & $3.18 \pm 0.66$ \\
150 & $10.7 \pm 3.33$ & $2.33 \pm 0.51$ & $3.20 \pm 0.81$ & $4.85 \pm 0.99$ \\
200 & $11.1 \pm 2.81$ & $1.78 \pm 0.38$ & $3.06 \pm 0.95$ & $6.21 \pm 1.30$ \\
300 & $10.2 \pm 2.27$ & $1.01 \pm 0.26$ & $3.79 \pm 0.85$ & $11.8 \pm 2.37$ \\
\hline \hline
\end{tabular}

we could determine only single and double ionization cross sections. Similarly for $\mathrm{Ar}$ and $\mathrm{Kr}$ we observed the $q=4$ peak (and even the $q=5$ peak for $\mathrm{Kr}$ ), but the counting statistics of the spectra allowed only the determination of single, double, and triple ionization cross sections.

Also shown in Figs. 4-7 are the results of our CTMC and simplified nCTMC calculations. In both model we considered the following shells: $K$ shell of He; $L_{1}, L_{2,3}$ shells of Ne; $L_{1}$, $L_{2,3}, M_{1}, M_{2,3}$ shells of Ar; $M_{1}, M_{2,3}, M_{4,5}, N_{1}, N_{2,3}$ shells

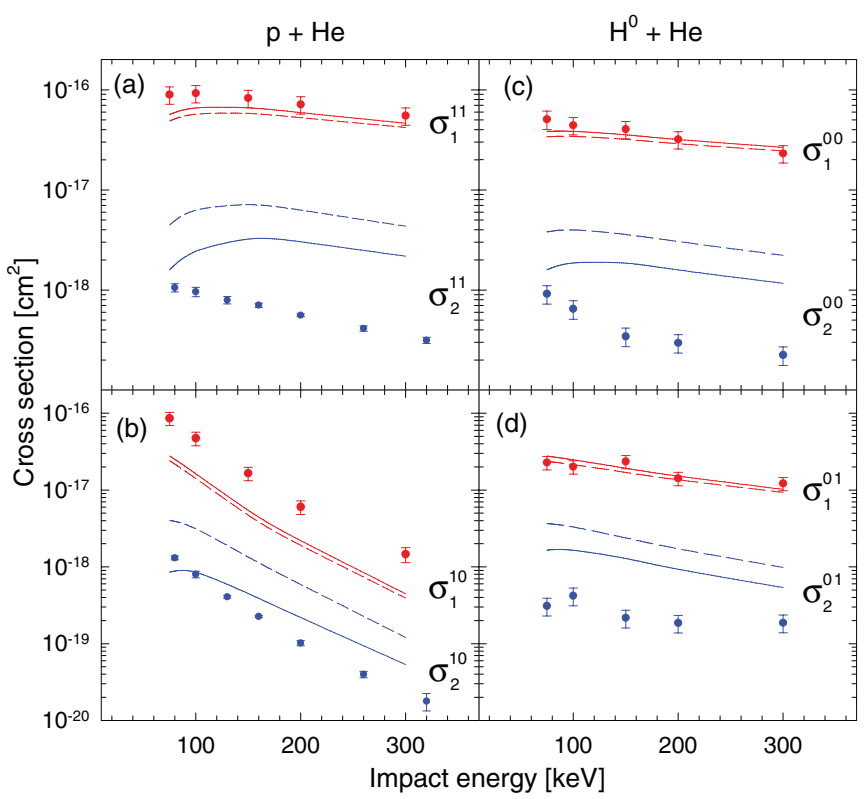

FIG. 4. (Color online) Cross sections for single and double ionization of $\mathrm{He}$. The notations: circles with error bars, experimental data; solid line, nCTMC; dashed line, CTMC. Panel (a): pure single $\left(\sigma_{1}^{11}\right)$ and double $\left(\sigma_{2}^{11}\right)$ ionization of the target by proton impact. Panel (b): pure electron capture $\left(\sigma_{1}^{10}\right)$ and electron capture with simultaneous target ionization $\left(\sigma_{2}^{10}\right)$ by proton impact. Panel (c): pure single $\left(\sigma_{1}^{00}\right)$ and double $\left(\sigma_{2}^{00}\right)$ ionization of the target by $\mathrm{H}^{0}$ impact. Panel (d): single $\left(\sigma_{1}^{01}\right)$ and double $\left(\sigma_{2}^{01}\right)$ ionization of the target by $\mathrm{H}^{0}$ impact with simultaneous ionization of the projectile. The single ionization data are results of the present work, the double ionization data in this figure (i.e., only for $\mathrm{He}$ ) are taken from the literature (Refs. [18,29], see text).

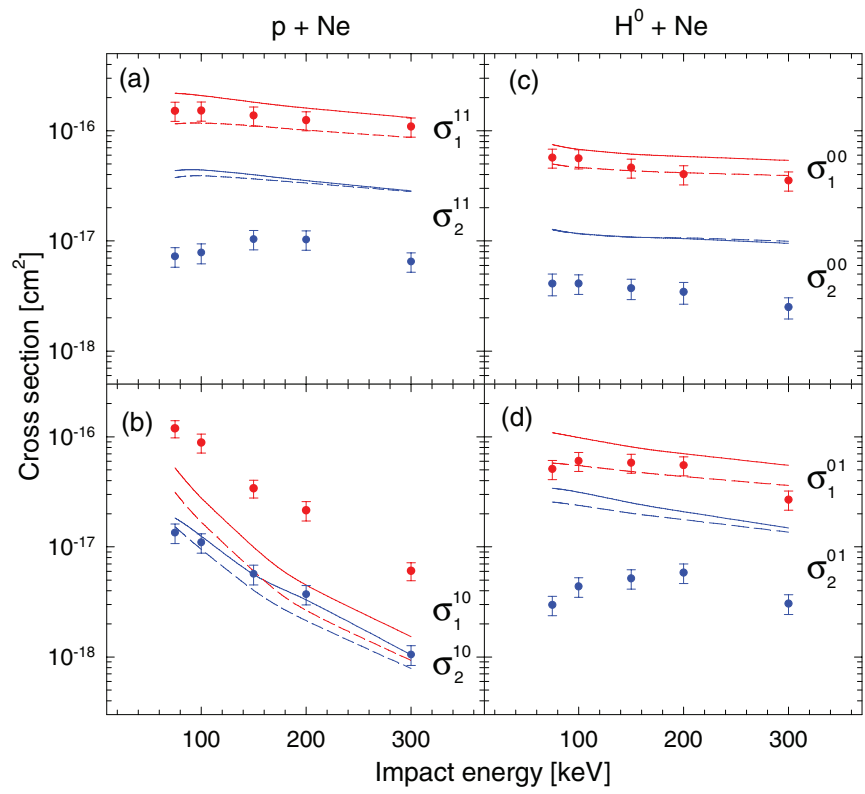

FIG. 5. (Color online) The same as Fig. 4 but for Ne target.

of $\mathrm{Kr}$. The contributions of the innermost shells ( $K$ shell of Ne and $\mathrm{Ar} ; K, L_{1}, L_{2,3}$ shell of $\mathrm{Kr}$ ) to the cross sections were negligibly small. For the inclusion of the relaxation effects following the primary vacancy creation (see Sec. III) we used the branching ratios $F_{\mu, k}$ tabulated in their paper by Montanari et al. [27]. The $F_{\mu, k}$ values have been determined on the basis of the results of photoionization experiments, and their use is justified by the fact that the photoionization of a given shell practically produces only a single initial vacancy.

From the comparison of the experimental data and the theoretical predictions we may draw the following conclusions, generally for all the targets. Both CTMC models correctly reproduce the slow energy dependence of the single pure

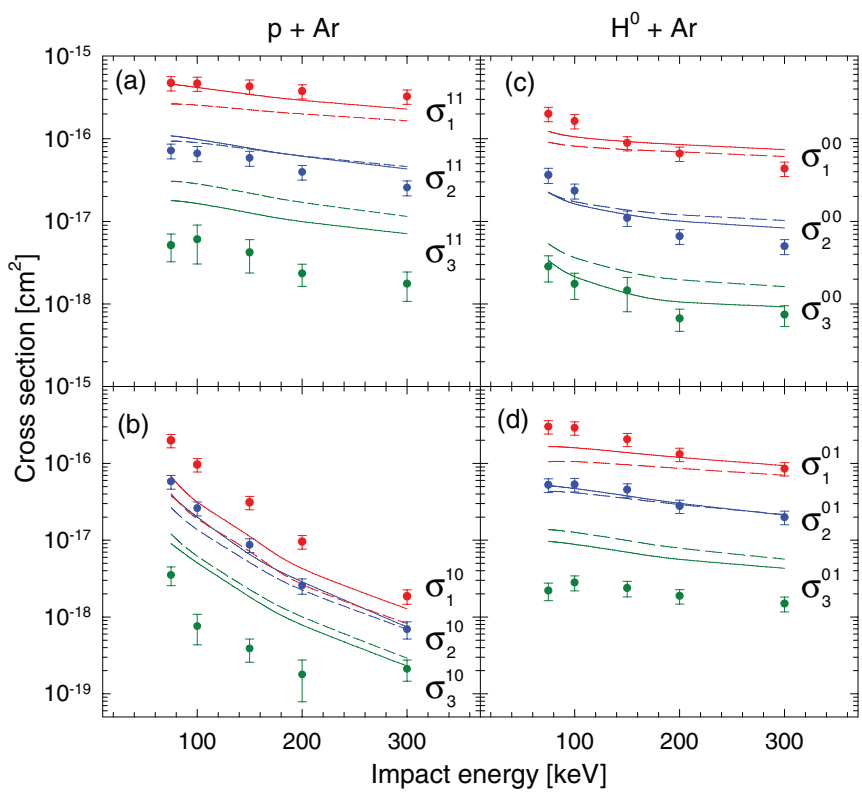

FIG. 6. (Color online) Cross sections for single, double, and triple ionization of Ar. For the notations of the data points, the theoretical curves, and the explanation of the panels (a)-(d) see Fig. 4. 


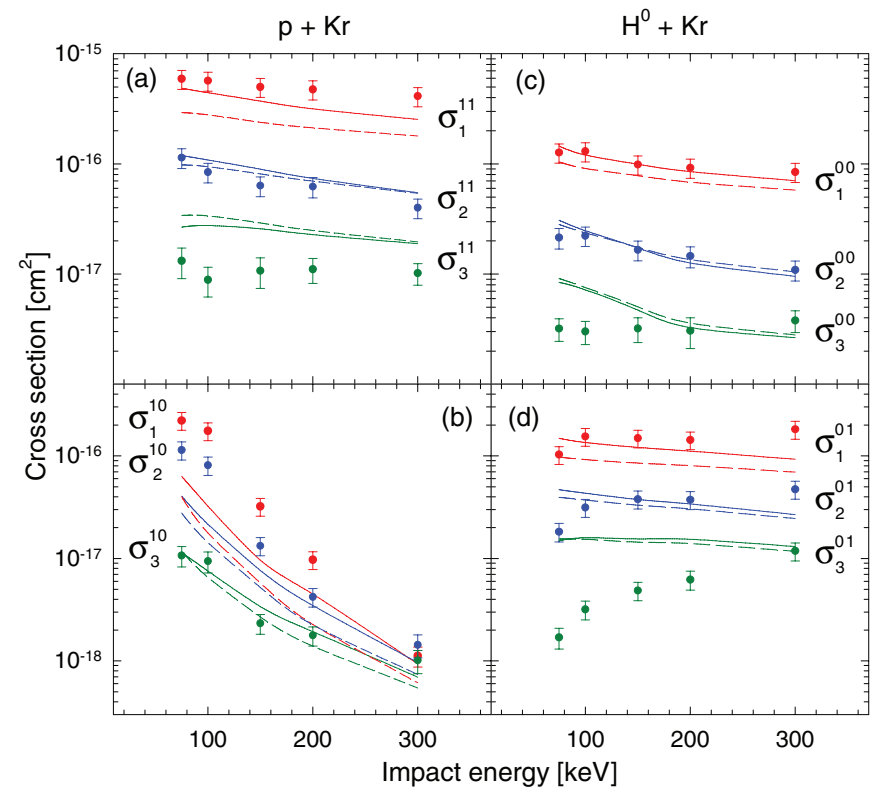

FIG. 7. (Color online) The same as Fig. 6 but for $\mathrm{Kr}$ target.

ionization cross sections for both projectiles. The same holds for the single ionization by $\mathrm{H}^{0}$ impact with simultaneous ionization of the projectile. The inclusion of the many-electron effects in nCTMC generally improves the agreement with the experiment, and we may conclude that the latter theory provides a reasonable description of the single pure ionization. For proton impact the steep decrease of the electron capture cross sections with increasing energy (without and with production of additional vacancies in the target) is also well described by both theories, however, there is a strong disagreement in magnitude.

Concerning the double pure ionization, for $\mathrm{Ar}$ and $\mathrm{Kr}$ the agreement between the experiment and the CTMC calculations is slightly worse but still acceptable, whereas both theories completely fail in this case for $\mathrm{He}$ and $\mathrm{Ne}$. The disagreement is particularly large for He. Interestingly, CTMC and nCTMC predict almost identical cross sections for the double pure ionization for $\mathrm{Ne}, \mathrm{Ar}$, and $\mathrm{Kr}$. For He the correction due to nCTMC is large and has good direction, but is still not enough.

For triple pure ionization a good agreement between the experiment and theory (nCTMC) can be observed only for $\mathrm{H}^{0}$ on $\mathrm{Ar}$ collisions. For $\mathrm{H}^{0}$ on $\mathrm{Kr}$ collision the agreement is also good at high impact energies, but the measured data do not follow the steep increase of the theoretical cross sections below $200 \mathrm{keV}$. In contrast to this energy dependence, for the same collision system the measured triple ionization cross sections with simultaneous ionization of the projectile steeply decrease with decreasing energy, whereas the calculated cross sections are almost constant over the whole energy range. For proton impact the measured triple pure ionization cross sections are strongly overestimated by the theories for both $\mathrm{Ar}$ and $\mathrm{Kr}$.

We call the attention to an interesting effect occurring in the energy dependence of the electron capture cross sections for proton on $\mathrm{Kr}$ collisions. The differences between the cross sections $q=1,2$, and 3 decrease with increasing impact energy. At $300 \mathrm{keV}$ the differences practically vanish; the double ionization cross section is even slightly higher than the single ionization one $\left(\sigma_{2}^{10} / \sigma_{1}^{10}=1.28 \pm 0.16\right.$ and $\sigma_{3}^{10} / \sigma_{1}^{10}=0.90 \pm 0.12$.) This effect can be traced back to the increasing role of the electron capture from the inner shells and by the fact that the Auger cascade following the ionization of the inner shells leads to additional vacancies in the outer shells. The $\mathrm{nCTMC}$ theory gives a qualitative account of the effect.

In the following we analyze the role played by the electron of $\mathrm{H}^{0}$ in the process of multiple ionization. To this we compare suitably chosen cross sections measured at proton and $\mathrm{H}^{0}$ impact. At a first glance one would assume that the pure ionization cross sections are the most suitable quantities for such a comparison. We show that this is not so. Let us consider, for example, the probability of the single pure ionization of an atom in one of its shells having $N$ electron by proton impacts [see Eq. (2)]:

$$
P_{i^{1} c^{0}}=N p_{i}\left(1-p_{i}-p_{c}\right)^{N-1} .
$$

The corresponding expression for $\mathrm{H}^{0}$ impact [see Eqs. (10) and (12)]:

$$
P_{i^{1}}^{00}=\left(1-P_{\mathrm{EL}}\right) N p_{i}^{0}\left(1-p_{i}^{0}\right)^{N-1} .
$$

The superscripts in $P_{i^{1}}^{00}$ denote the initial and final charge state of $\mathrm{H}^{0}$. In Eqs. (17) and (18) $p_{i}$ and $p_{i}^{0}$ are the singleelectron ionization probabilities for proton and $\mathrm{H}^{0}$ impact, respectively. The role of the electron in $\mathrm{H}^{0}$ is manifested by the relation between $p_{i}$ and $p_{i}^{0}$. This means that those processes are comparable the probabilities of which depend on $p_{i}$ and $p_{i}^{0}$ in the same way. As is seen, the structure of the above expressions is quite different: $P_{i^{1} c^{0}}$ depends also on the electron capture probability $p_{c}$, and the expression of $P_{i^{1}}^{00}$ contains the electron loss probability $P_{\mathrm{EL}}$.

Better candidates for the comparison are the ionization cross sections that are inclusive with respect to processes other than pure ionization, i.e., $\sum_{j} \sigma_{q}^{1 j}$ for proton impact (summation over the electron capture channel) and $\sum_{j} \sigma_{q}^{0 j}$ for $\mathrm{H}^{0}$ impact (summation over the electron loss channel). To see this, let us consider the sum of the corresponding ionization probabilities. It is easy to show that for proton impact

$$
\begin{aligned}
\sum_{m=0}^{N-n} P_{i^{n} c^{m}} & =\sum_{m=0}^{N-n}\left(\begin{array}{l}
N \\
n
\end{array}\right)\left(\begin{array}{c}
N-n \\
m
\end{array}\right) p_{i}^{n} p_{c}^{m} p_{e}^{N-(n+m)} \\
& =\left(\begin{array}{l}
N \\
n
\end{array}\right) p_{i}^{n}\left(1-p_{i}\right)^{N-n} .
\end{aligned}
$$

For $\mathrm{H}^{0}$ impact the sum of the probabilities for the ionization of the target without and with the simultaneous ionization of the projectile leads to the same dependence on $p_{i}^{0}$ as that on $p_{i}$ seen in Eq. (19):

$$
\begin{aligned}
P_{i^{n}}^{00}+P_{i^{n}}^{01}= & \left(1-P_{\mathrm{EL}}\right)\left(\begin{array}{l}
N \\
n
\end{array}\right) p_{i}^{0^{n}}\left(1-p_{i}^{0}\right)^{N-n} \\
& +P_{\mathrm{EL}}\left(\begin{array}{l}
N \\
n
\end{array}\right) p_{i}^{0^{n}}\left(1-p_{i}^{0}\right)^{N-n} \\
= & \left(\begin{array}{l}
N \\
n
\end{array}\right) p_{i}^{0^{n}}\left(1-p_{i}^{0}\right)^{N-n} .
\end{aligned}
$$




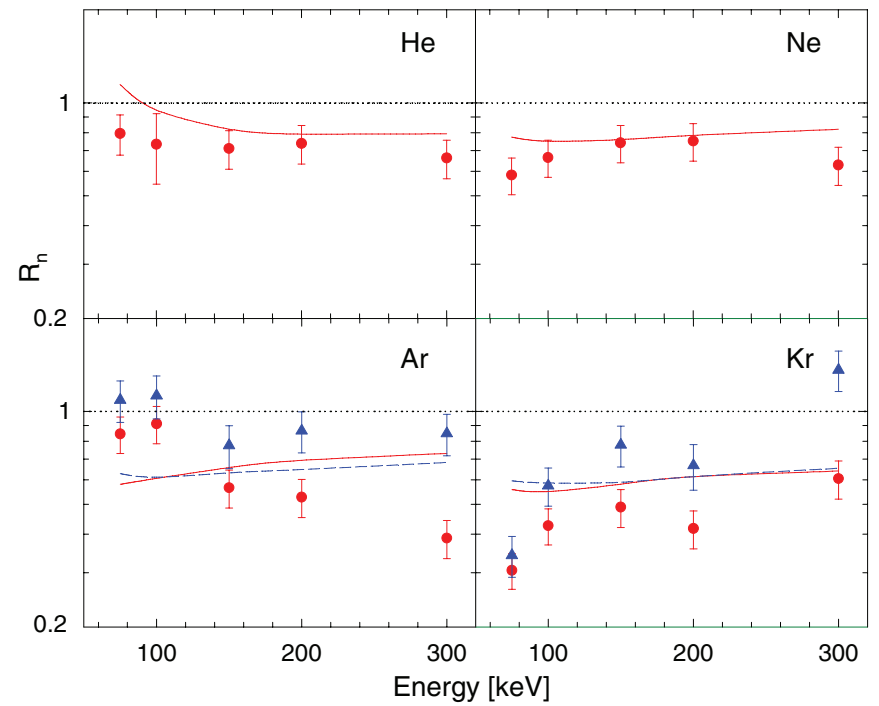

FIG. 8. (Color online) The measured and calculated $R_{n}$ ratios of the inclusive pure ionization cross sections for $\mathrm{H}^{0}$ and proton impact [for the definition of $R_{n}$ see Eq. (21)]. The notations are as follows. Experiment: circles, $n=1$; triangles, $n=2$. Theory (nCTMC): solid line, $n=1$; dashed line, $n=2$.

On the basis of the above arguments we tried to extract some information about the role played by the electron of the $\mathrm{H}^{0}$ projectile by comparing our measured and calculated inclusive cross sections for $\mathrm{H}^{0}$ and proton impact. To this we determined the following ratios:

$$
R_{n}=\frac{\sigma_{n}^{00}+\sigma_{n}^{01}}{\sigma_{n}^{11}+\sigma_{n+1}^{10}} .
$$

Here $n$ is the degree of the pure (direct) ionization of the target atom.

The obtained $R_{n}$ ratios $(n=1,2)$ are plotted in Fig. 8. For $n=1$ all the measured data are below 1, i.e., the inclusive pure single ionization cross sections for $\mathrm{H}^{0}$ impact are smaller than those for proton impact. For $n=2$ the data are somewhat larger, but most of them lie also below 1. Except for the lowest energy point for $\mathrm{He}$, the $R_{n}$ ratios predicted by nCTMC are also smaller than 1 . The agreement between experiment and theory is reasonable for $\mathrm{He}$ and $\mathrm{Ne}$. Although the data for $\mathrm{Ar}$ and $\mathrm{Kr}$ scatter widely, their average value of about $0.5-0.6$ is in agreement with the prediction of the theory. The fact that with a few exceptions all the experimental and the theoretical $R_{n}$ ratios are smaller than 1 suggests that the dominant role of the electron in $\mathrm{H}^{0}$ is the screening effect exerted on the Coulomb field of the proton. For He at lower impact energies nCTMC predicts also antiscreening, but the experimental data do not support it.

The finding $R_{n}<1$ means that the quasifree electron scattering model fails for the collisions studied in the present work. Although the regarded energy range is high enough for the ionization of outer shells of the target atoms by the electron of $\mathrm{H}^{0}$ (see Sec. I), in this model $R_{n}>1$ is expected because of the incoherent sum of the contributions of the proton and the electron constituent of $\mathrm{H}^{0}$. Of course, this does not mean that we can exclude the possibility of the active role of the projectile electron. In a more complete, realistic quantum mechanical description the contributions of the proton and the electron have to be added coherently, and a destructive interference between the amplitudes of the two processes may lead also to $R_{n}<1$.

\section{CONCLUSIONS}

Experimental and theoretical cross sections for the multiple ionization of $\mathrm{He}, \mathrm{Ne}, \mathrm{Ar}$, and $\mathrm{Kr}$ by proton and $\mathrm{H}^{0}$ impact have been presented in the energy range 75-300 keV. In the measurements the final charge states of both collision partners were observed. The theoretical cross sections were calculated using the standard CTMC method in the three-body approximation and a simplified version of the $\mathrm{nCTMC}$ model that takes into account the many-body character of the collision. A satisfactory description of the single ionization for both projectiles has been achieved by the latter theory; however, increasing deviation is observed between the predictions of both models and the experimental data with increasing number of created vacancies and with decreasing target atomic number.

The failure of both CTMC models in reproduction of the measured cross sections for higher recoil target charge states can be traced back to the fact that CTMC, as a classical theory, cannot provide accurate single-electron ionization, capture, and loss probabilities as a function of the impact parameter. Although satisfactory single ionization cross sections can be determined even with less accurate probability functions, for the determination of double, triple, etc. ionization cross sections the error due to the inaccuracies increases with power of the multiplicity. It is an interesting question how much improvement can be achieved in the description of the multiple ionization data with use of more accurate single-electron probability functions provided by quantum mechanical ionization theories. Such calculations would shed light also on the possible presence of electron correlation effects, i.e., the limit of the application of the independent particle model.

As far as the role played by the electron of the $\mathrm{H}^{0}$ projectile in the multiple vacancy creation is concerned, the comparison of suitably chosen measured cross sections for the two projectiles shows that the $\mathrm{H}^{0}$ projectile on average is $20 \%-50 \%$ less effective in creation of vacancies than the proton. The nCTMC model is in accordance with this finding, which indicates that the projectile electron dominantly plays a passive screening role in the collision. However, in the quantum mechanical view of the ionization process the active role of the electron (excitation or ionization of the target electrons) also cannot be excluded, because destructive interference between the ionization amplitude of the electron and proton constituents of $\mathrm{H}^{0}$ may also reduce the cross section.

\section{ACKNOWLEDGMENTS}

This work was supported by the National Information Infrastructure Program (NIIF), the National Scientific Research Foundation, the National Office for Research and Technology of Hungary (NKTH-OTKA, Grant No. K67719), and the TÁMOP-4.2.2/B-10/1-2010-0024 project (cofinanced by EU and the European Social Fund). 
[1] C. C. Montanari and J. E. Miraglia, J. Phys. B 45, 105201 (2012).

[2] J. H. McGuire, N. Stolterfoht, and P. R. Simony, Phys. Rev. A 24, 97 (1981).

[3] L. Végh and L. Sarkadi, J. Phys. B 16, L727 (1983).

[4] P. Richard, D. H. Lee, T. J. M. Zouros, J. M. Sanders, and J. L. Shinpaugh, J. Phys. B 23, L213 (1990).

[5] K. Taulbjerg, J. Phys. B 23, L761 (1990).

[6] D. Brandt, Phys. Rev. A 27, 1314 (1983).

[7] J. A. Tanis et al., Nucl. Instrum. Methods B 10/11, 128 (1985).

[8] S. Brawley, S. Armitage, J. Beale, D. Leslie, A. Williams, and G. Laricchia, Science 330, 789 (2010).

[9] S. J. Brawley, A. I. Williams, M. Shipman, and G. Laricchia, Phys. Rev. Lett. 105, 263401 (2010).

[10] R. Anholt, Phys. Lett. A 114, 126 (1986).

[11] E. C. Montenegro, W. S. Melo, W. E. Meyerhof, and A. G. de Pinho, Phys. Rev. Lett. 69, 3033 (1992).

[12] B. Najjari and A. B. Voitkiv, Phys. Rev. A 87, 034701 (2013).

[13] R. K. Janev, in Nuclear Fusion Research, Springer Series in Chemical Physics Vol. 78, edited by R. E. H. Clark and D. Reiter (Springer, Berlin, 2005), pp. 415-435.

[14] V. V. Afrosimov, Ya. A. Mamaev, M. N. Panov, and N. V. Fedorenko, Sov. Phys. JETP 28, 52 (1969).

[15] E. Horsdal Pedersen and L. Larsen, J. Phys. B 12, 4099 (1979).
[16] L. H. Andersen, L. B. Nielsen, and J. Sørensen, J. Phys. B 21, 1587 (1988).

[17] R. D. DuBois and L. H. Toburen, Phys. Rev. A 38, 3960 (1988).

[18] R. D. DuBois and Á. Kövér, Phys. Rev. A 40, 3605 (1989).

[19] L. Víkor, L. Sarkadi, F. Penent, A. Báder, and J. Pálinkás, Phys. Rev. A 54, 2161 (1996).

[20] A. Báder, L. Sarkadi, Gy. Hegyesi, L. Víkor, and J. Pálinkás, Meas. Sci. Technol. 6, 959 (1995).

[21] R. Abrines and I. C. Percival, Proc. Phys. Soc. London 88, 861 (1966).

[22] R. E. Olson and A. Salop, Phys. Rev. A 16, 531 (1977).

[23] B. Sulik and K. Tőkési, Adv. Quantum Chem. 52, 253 (2007).

[24] A. E. S. Green, D. L. Sellin, and A. S. Zachor, Phys. Rev. 184, 1 (1969).

[25] R. H. Garvey, C. H. Jackman, and A. E. S. Green, Phys. Rev. A 12, 1144 (1975).

[26] C. O. Reinhold and C. A. Falcón, Phys. Rev. A 33, 3859 (1986).

[27] C. C. Montanari, E. C. Montenegro, and J. E. Miraglia, J. Phys. B 43, 165201 (2010).

[28] R. E. Olson, J. Ullrich, and H. Schmidt-Böcking, J. Phys. B 20, L809 (1987).

[29] M. B. Shah and H. B. Gilbody, J. Phys. B 18, 899 (1985).

[30] R. D. DuBois and S. T. Manson, Phys. Rev. A 35, 2007 (1987). 